\title{
Hot-electron intraband luminescence from single hot spots in noble-metal nanoparticle
}

\section{films}

\author{
Tobias Haug, Philippe Klemm, Sebastian Bange*), John M. Lupton
}

Institut für Experimentelle und Angewandte Physik, Universität Regensburg, 93051

Regensburg, Germany

Disordered noble-metal nanoparticle films exhibit highly localized and stable non-linear light emission from sub-diffraction regions upon illumination by near-infrared femtosecond pulses. Such hot spot emission spans a continuum in the visible and near-infrared spectral range. Strong plasmonic enhancement of light-matter interaction and the resulting complexity of experimental observations have prevented the development of a universal understanding of the origin of light emission. Here, we study the dependence of emission spectra on excitation irradiance and provide the most direct evidence yet that the continuum emission observed from both silver and gold nanoparticle aggregate surfaces is caused by recombination of hot electrons within the conduction band. The electron gas in the emiting particles, which is effectively decoupled from the lattice temperature for the duration of emission, reaches effective temperatures of several thousand Kelvin and acts as a sub-diffraction incandescent light source on sub-picosecond time scales.

PACS numbers:

78.67.Bf Optical properties of low-dimensional, mesoscopic, and nanoscale materials and structures - Nanocrystals and nanoparticles 73.20.Mf Collective excitations including excitons, polarons, plasmons and other 
charge-oscillations

78.47.db Spectroscopy of solid state dynamics - conduction electrons

78.55.Hx Photoluminescence of other solid inorganic materials

78.60.Lc Optically stimulated luminescence

Metals appear shiny because they reflect light incident on them under conservation of energy and momentum, only a fraction of photons being lost to absorption through the excitation of damped surface plasmons. In addition, luminescence can occur by electron-hole recombination, although quantum yields for radiative emission are typically extremely low, in the range of $10^{-6}$. Such luminescence induced by laser irradiation has been known since the late 1960s [1], and continues to intrigue. Upon ultraviolet irradiation, luminescence of gold and silver surfaces is linear in excitation power and arises from the radiative recombination of $d$-band holes with relaxed $s p$-band electrons [2]. Under infrared excitation, non-linear up-converted broadband emission has been reported as background in surface-enhanced harmonic generation on rough silver surfaces [3] and was later shown to be spatially localized to regions of sub-diffraction hot spots [4]. With the excitation power-dependence being close to quadratic, this phenomenon has been mostly discussed in the context of conduction-band electrons recombining with $d$-band holes following two-photon absorption [2,5]. However, the wide interest in the plasmonic properties of metal nanoparticles in general and surface-enhanced spectroscopies on nanoparticle-decorated surfaces in particular has led to renewed scrutiny of the origins of nonlinear luminescence. While surface non-linear mixing in the presence of strong plasmonenhanced electric fields has been discussed as a mechanism for the generation of up-converted broadband emission [6], it is difficult to reconcile with the observed similarity between individual hot spot emission spectra [7] and their insensitivity to excitation wavelength [4], dielectric coating [8] and photoinduced surface charging effects [9]. Moreover, emission 
intensity does not depend on pulse length up to $1 \mathrm{ps}$ in duration, but decreases above $1 \mathrm{ps}$ [10], pointing to a sequential excitation process affected by electron-phonon coupling. The emission source neither coincides with Raman-active plasmonic hot spot sites [11], nor is it sensitive to surface effects [9], further suggesting a volumetric mechanism. The emission spectrum does not systematically depend on surface coverage and particle density, once interference effects are accounted for $[7,12]$. In addition, although silver and gold have very different interband transition energies, nanoparticle aggregates can exhibit similar broadband emission $[7,13]$. This similarity casts doubt on the notion that multiphoton-excited interband recombination events are the primary source of up-converted continuum emission. Furthermore, one-photon excited interband luminescence is known to have a finite lifetime (tens of picoseconds) and exhibit blinking on the level of single hot spots, whereas non-linear continuum emission has a lifetime shorter than a few picoseconds and does not blink [7]. Finally, up-converted continuum emission is accompanied by down-converted radiation in the infrared spectral range above the excitation wavelength, i.e. for photon energies far below those of interband transitions. This down-converted (Stokes) emission has not been studied extensively, but was found to follow a linear excitation power dependence. It was accordingly attributed to intraband luminescence, i.e. luminescent electronic transitions within the conduction band [13]. Although negligible on smooth metal surfaces, the presumed breakdown of symmetry and momentum selection rules in nanoscopic metal particles is expected to allow efficient luminescent recombination $[13,14]$. Thus far, no unified physical understanding of the emission phenomenon has emerged that covers luminescence from silver and gold surfaces under pulsed infrared excitation, both in the up-conversion and down-conversion regime.

Metal surfaces interact with infrared laser radiation mainly by collective electron plasma oscillations. These collective states dephase into single electron excitations on timescales of a 
few femtoseconds [15], which in turn scatter over a timescale of $\sim 100$ fs to establish a FermiDirac distribution that can reach effective temperatures of several thousand Kelvin [16,17]. Given that electron-phonon scattering dissipates this excess energy within picoseconds, only select studies have actually considered the role of hot electrons in the context of radiative recombination, either by interband recombination [18] or through resonance luminescence [19]. Although luminescent intraband transitions are known from few-atom metal clusters [20], there is, as yet, no unequivocal proof that such an effect also arises in metal nanoparticles and rough metal surfaces.

The present work provides direct spectroscopic evidence for luminescent intraband transitions in a hot electron gas and is guided by three principles. First, only studies of single emission sites can identify spectroscopic signatures of a heated electron gas in metal nanoparticles, since a priori deconvolution of a non-linear system response from an inhomogeneously distributed ensemble of hot spots is impossible. Second, the emission spectrum is subject to the antenna properties of the local plasmonic environment controlling the photonic density of states [21]. To test physical models of the luminescence mechanism, we therefore only rely on relative spectral changes in emission upon modification of excitation parameters. Third, possible contributions of interband luminescence need to be excluded. For silver, the observed luminescence is further removed from the interband transition than for gold (transitions occur at $\sim 4.0 \mathrm{eV}$ and $\sim 2.4 \mathrm{eV}$, respectively [22]): we therefore discuss luminescence from silver first, extending the results to gold after the emission mechanism is identified.

Densely packed random silver particle films formed by the Tollens reaction exhibit continuum emission hot spots at a spatial density low enough to enable single-site spectroscopy [4]. Compared to isolated metal nanoparticles, the excitation cross-section of single emitter sites is 
enhanced through coupling between individual particles [12]. This effective coupling explains why non-linear continuum emission from silver has so far only been reported from nanoparticle films and not from isolated particles. We prepared silver nanoparticle films as described previously (see Ref. [7] for characteristic electron micrographs), mounted these under a vacuum of $10^{-6} \mathrm{mbar}$ and illuminated them with a weakly focused laser beam $(70 \mu \mathrm{m}$ spot diameter). Luminescence was collected by a microscope objective and detected by a CMOS or CCD camera, optionally passed through a grating spectrometer. Figure 1 shows the spatial emission distribution upon wide-field excitation with 86 fs long laser pulses at $80 \mathrm{MHz}$ repetition rate and a wavelength of $770 \mathrm{~nm}$. The luminescence spectrum of a single emissive site covers a wide wavelength range from $0.4 \mu \mathrm{m}$ to above $1 \mu \mathrm{m}$. The spectrum is disrupted around $770 \mathrm{~nm}$ by a transmission filter used to attenuate scattered laser light. The laser spectrum is superimposed in the graph. The non-linearity in emission is commonly characterized by measuring the emitted photon flux $\phi$ as a function of excitation irradiance $E$, as shown in panel (c). We differentiate between up-converted (blue) and down-converted (red) emission. The intensity dependence is characterized by fitting a linear relationship $\log (\phi)=$ const. $+p \cdot \log (E)$ to a doublelogarithmic representation of the measured $\phi$ and $E$, where the slope $p$ represents the nonlinear power-law exponent. For the single emitter in Fig. 1, the emission intensity integrated over wavelengths below $723 \mathrm{~nm}$ (i.e. the blue curve) is characterized by an exponent of $p=$ $2.04 \pm 0.09$, whereas the down-converted emission (above $818 \mathrm{~nm}$ ) is described by $p=1.22 \pm$ 0.06). These values have conventionally been attributed to two-photon and one-photon excitation of luminescence, respectively [13].

Such a spectrally integrated analysis of the excitation irradiance dependence, however, does not faithfully convey the information available from the experiment. Figure 2 a compares emission spectra for both low and high irradiance on a logarithmic scale, for both pulsed and 
c.w. excitation. Both down-converted and up-converted emission spanning the visible spectrum is observed under pulsed excitation, whereas under c.w. illumination only weak downconverted emission is detected. Discrete bands above and below the excitation wavelength are attributed to Stokes and anti-Stokes Raman scattering, respectively. Under pulsed excitation, the emission blue-shifts at higher irradiance, as observed in Fig. 2a by the shift of the peak photon flux to higher energies. This shift indicates a spectral variation of the power-law exponent $p(v)$, when determined as a function of the emitted photon frequency $v$. Figure $2 b$ shows $p(v)$ as extracted from the irradiance dependence of the emission at each frequency. Under c.w. excitation (red data points) $p(v) \approx 1$, i.e. constant. Pulsed excitation (black) is instead characterized by a frequency-dependent exponent that not only suggests a linear relation, but is indeed consistent with a direct proportionality $p(v) \propto v$ as data points can be extrapolated to $p \rightarrow 0$ for $v \rightarrow 0$. A physical model for non-linear emission must explain this telltale proportionality. We argue below that this is a straightforward property of intraband recombination of hot carriers.

Following absorption of a femtosecond laser pulse by the electrons in a metal nanoparticle, electron-phonon coupling leads to a time-dependent electron-gas temperature that has conventionally been described by a rate equation model with two temperatures [19]. For the present purpose, we simply attribute a fixed effective temperature $T_{\mathrm{e}} \gg T$ to the electron system for the timescale of the luminescence, with $T$ being the lattice temperature of the particle. A general form $T_{\mathrm{e}}^{a} \propto E$ of the excitation irradiance dependence of $T_{\mathrm{e}}$ is assumed - for bulk metals $a=2$ is well known from the heat capacity of a free electron gas [23]. Strong plasmonic confinement of the electric fields and small dimensions of the emitting particle (which gives rise to large wavevector uncertainty) can presumably relax symmetry selection and momentum conservation rules to allow for intraband dipole transitions which would 
otherwise be forbidden in the bulk [13]. In the limit $h v \gg k_{\mathrm{B}} T_{\mathrm{e}}$, the spontaneous radiative transition rate of Fermi-Dirac distributed conduction-band electrons takes a simple Boltzmann shape $\phi(v)=f(v) \exp \left(-h v / k_{\mathrm{B}} T_{\mathrm{e}}\right)$, where $h$ and $k_{\mathrm{B}}$ are the Planck and Boltzmann constant, respectively (see Supplemental Material and Fig. S1 for details [24]). $f(v)$ is a function of the photonic mode density and thus changes with the plasmonic environment, but is independent of $T_{\mathrm{e}}$ and irradiance $E$. Experimentally, $\phi(E)$ follows a power-law as seen in Fig. 2b. Since the irradiance $E$ is usually not varied by more than a factor of 4 around a reference value $E^{*}$, the temperature is limited to a narrow range around a corresponding value $T_{\mathrm{e}}^{*}$. In normalized logarithmic representation, using $\left(T_{\mathrm{e}} / T_{\mathrm{e}}^{*}\right)^{a}=E / E^{*}$, the emitted photon flux is

$$
\log (\phi)=\log [f(v)]-\frac{h v}{k_{\mathrm{B}} T_{\mathrm{e}}^{*}} \exp \left[-\frac{1}{a} \log \left(\frac{E}{E^{*}}\right)\right]
$$

Indeed, in the limited irradiance range, the exponential term can be approximated linearly to good precision as

$$
\log (\phi)=g\left(v, T_{\mathrm{e}}^{*}\right)+\frac{h v}{a l_{\mathrm{B}} T_{\mathrm{e}}^{*}} \log \left(\frac{E}{E^{*}}\right),
$$

where $g=\log (f)-h v / k_{\mathrm{B}} T_{\mathrm{e}}^{*}$ is independent of $E$. Eq. 2 describes a power-law type behavior $\phi \propto E^{p}$ with exponent $p(v)=h v / a k_{\mathrm{B}} T_{\mathrm{e}}^{*}$, where both $a$ and $T_{\mathrm{e}}^{*}$ depend on the thermodynamic properties of the nanoscale electron system and the effective excitation cross section as well as the experimentally chosen mean irradiance $E^{*}$. The linearity of $p(v)$ found in Fig. 2 is therefore expected for excitation conditions that deposit sufficient energy in the electron gas such that luminescence occurs before the onset of cooling by electron-phonon scattering. In the limit of c.w. excitation, electron heating is suppressed by phonon scattering and $T_{\mathrm{e}}$ equals the lattice temperature. In this case, emission stems from transitions involving electrons much closer to the Fermi edge and is linear in excitation power $(p=1)[25]$. This situation is described by the red data points in Fig. $2 b$. 
The appearance of localized, broadband emission hot spots upon femtosecond laser excitation of a rough metal surface is mostly insensitive to preparation conditions, and the phenomenon is not limited to silver. Non-linear hot-spot luminescence from gold surfaces is generally found to follow the same behavior. Figure 3 shows a typical emission spectrum and corresponding non-linear power exponents measured for a single hot spot on a rough gold film evaporated on top of a glass substrate. Owing to the smaller interband transition energy compared to silver, the high-energy side of the spectrum exhibits increased damping. Nonetheless, the linearity of $p(v)$ is clearly reproduced except for frequencies with strong Raman contributions around $1.45 \mathrm{eV}$ and $1.79 \mathrm{eV}$. The spectral shape of the gold hot spot emission is remarkably similar to that of silver film hot spots, despite the large difference in interband transition energies. It was previously surmised that down-converted luminescence of gold nanostructures arises from intraband transitions of photoexcited electrons in a distribution in thermal equilibrium with the lattice (i.e. $p(v)=1$ ), whereas up-converted emission was thought to stem from conventional two-photon excitation of the interband transition (i.e. $p(v)=2$ ) [13]. This is clearly not so, given the linear variation of $p(v)$. Luminescence from gold nanostructures under femtosecond excitation thus arises from the same mechanism as that in silver: intraband transitions of a hot electron gas.

While from equ. (2) parameters $a$ and $T_{\mathrm{e}}^{*}$ cannot be determined independently, the analysis suggests that it should be possible to observe a subtle change of exponent $p$ with irradiance $E^{*}$ since $T_{\mathrm{e}}^{*} \propto\left(E^{*}\right)^{1 / a}$. Separation of $a$ and $T_{\mathrm{e}}^{*}$ is possible by measuring $p$ for a fixed emission frequency $v$ as a function of the mean irradiance $E^{*}$. Figure 4a shows two sweeps of emission as a function of irradiance on a single hot spot (not the same spot as examined in Fig. 2). The emission follows a power-law-like dependence, and can indeed be tracked over more than one 
order of magnitude change in irradiance with excellent repeatability. As shown in Fig. 4b, the power coefficient calculated from the local slope in panel (a) follows a linear relationship in double-logarithmic representation, as expected from $\log (p) \propto-a^{-1} \log \left(E^{*}\right)$. The exponent $a$ can therefore be determined by a linear fit. Figure 4c shows that this analysis can be repeated for the full spectral range, yielding an average exponent of $a=4.57$. Given excellent agreement of the model with experimental observations, the effect of irradiance $E^{*}$ on the temperature $T_{\mathrm{e}}^{*}$ of the electron gas is estimated from $T_{\mathrm{e}}^{*}=h v / a(v) k_{\mathrm{B}} p(v)$. As shown in Fig. $4 \mathrm{~d}$, the temperature values fall in the range of $2,000 \mathrm{~K}$ to $4,000 \mathrm{~K}$.

It may appear surprising to find a simple thermodynamic model reproducing the main experimental observations of a spectrally varying power-law exponent $p(v)$. However, while high electron gas temperatures explain the spectral bandwidth of the emission in general (Fig. 1b), no attempt is made here to discuss the spectral shape in detail, since it is modified strongly by the local photonic density of states which varies between different hot spots due to the heterogeneity of the particles and their environment [4]. Nonetheless, fitting a Planck-type black-body spectrum directly to a range of the emission yields an irradiance-dependent blackbody temperature that follows a power-law with an exponent of $a=4.2$, as shown in the Supplemental Material Fig. S2 [24], i.e. similar to that extracted in Fig. 4c from the irradiance dependence. The spectral shape of the emission is therefore consistent with black-body radiation. A survey of 122 single hot spots on the silver surface shows a broad distribution of $a$ values between 2 and 8 , with most values centered around $a=4$ (Supplemental Material Fig. S3 [24]). We note that the parameter $a$ is over twice as large as the temperature exponent for a free electron gas $a=2$ [23] and propose that the strong scatter in $a$ may arise from variations in the emissive particle size, which impacts the energy absorbed and thus electron-electron and electron-phonon scattering times $[16,26]$. 
While absorption of ultrashort laser pulses on metal surfaces is well-known to heat the electron gas through intraband transitions, their role in radiative recombination in metal nanoparticles has previously been largely overlooked. Although we can only speculate on the physical nature of the hot spot as being a small metal nanoparticle, single-site irradiance dependent spectroscopy uncovers straightforward indicators supporting the notion of luminescent intraband recombination in a hot electron distribution as modeled by a simple thermodynamic ansatz. This approach is crucial since the main finding of a linear $p(v)$ is not unambiguously resolved in ensemble spectroscopy, as shown in the Supplemental Material, Fig. S4 [24]. Luminescence effects on disordered metal surfaces are orders of magnitude stronger than for dilute ensembles of metal nanoparticles, or for smooth metal films, as the plasmonic environment of the nanoparticle distribution effectively increases the available excitation crosssection by scattering and interparticle coupling [12]. At the same time, depending on the surface fabrication method, emitter densities can be low enough to enable single-site spectroscopy. Continuum-emission hot spots effectively constitute ultrafast incandescent broadband light sources that enable nanoscopic pump-probe spectroscopy, but also raise intriguing questions regarding photon statistics in emission, since photon bunching is expected. The spectroscopy of hot electrons in metal nanoparticle systems by considering their characteristic luminescence will be relevant to energy harvesting and photocatalysis [27,28], and provides a direct window to study phonon-bottleneck phenomena. 


\section{References:}

[1] A. Mooradian, Phys. Rev. Lett. 22, 185 (1969).

[2] G. T. Boyd, Z. H. Yu, and Y. R. Shen, Phys. Rev. B 33, 7923 (1986).

[3] C. K. Chen, A. R. B. de Castro, and Y. R. Shen, Phys. Rev. Lett. 46, 145 (1981).

[4] N. J. Borys, E. Shafran, and J. M. Lupton, Sci. Rep. 3, 2090 (2013).

[5] P. Zijlstra and M. Orrit, Rep. Prog. Phys. 74, 106401 (2011).

[6] S. Ducourtieux, S. Gresillon, A. C. Boccara, J. C. Rivoal, X. Quelin, P. Gadenne, V. P. Drachev, W. D. Bragg, V. P. Safonov, V. A. Podolskiy, Z. C. Ying, R. L. Armstrong, and V. M. Shalaev, J. Nonlinear Opt. Phys. Mater. 9, 105 (2000).

[7] N. J. Borys and J. M. Lupton, J. Phys. Chem. C 115, 13645 (2011).

[8] S. Nah, L. Li, and J. T. Fourkas, J. Phys. Chem. A 113, 4416 (2009).

[9] N. J. Borys, M. J. Walter, and J. M. Lupton, Phys. Rev. B 80, 161407 (2009).

[10] P. Biagioni, M. Celebrano, M. Savoini, G. Grancini, D. Brida, S. Mátéfi-Tempfli, M. Mátéfi-Tempfli, L. Duò, B. Hecht, G. Cerullo, and M. Finazzi, Phys. Rev. B 80, 045411 (2009).

[11] M. J. Walter, N. J. Borys, G. Gaefke, S. Höger, and J. M. Lupton, J. Am. Chem. Soc. 130, 16830 (2008).

[12] P. Klemm, T. Haug, S. Bange, and J. M. Lupton, Phys. Rev. Lett. 113, 266805 (2014).

[13] M. R. Beversluis, A. Bouhelier, and L. Novotny, Phys. Rev. B 68, 115433 (2003).

[14] T. P. Bigioni, R. L. Whetten, and O. Dag, J. Phys. Chem. B 104, 6983 (2000). 
[15] R. D. Muino, E. E. Krasovskii, W. Schattke, C. Lienau, and H. Petek, Electromagnetic interactions with solids in Dynamics at Solid State Surfaces and Interfaces: Volume 2: Fundamentals edited by U. Bovensiepen, H. Petek, and M. Wolf, Wiley-VCH, (2012).

[16] B. Y. Mueller and B. Rethfeld, Phys. Rev. B 87, 035139 (2013).

[17] M. Perner, P. Bost, U. Lemmer, G. von Plessen, J. Feldmann, U. Becker, M. Mennig, M. Schmitt, and H. Schmidt, Phys. Rev. Lett. 78, 2192 (1997).

[18] D. S. Sitnikov, A. A. Yurkevich, M. S. Kotelev, M. Ziangirova, O. V. Chefonov, I. V. Ilina, V. A. Vinokurov, A. V. Muradov, I. Itzkan, M. B. Agranat, and L. T. Perelman, Laser Phys. Lett. 11, 075902 (2014).

[19] J. Y. Huang, W. Wang, C. J. Murphy, and D. G. Cahill, Proc. Natl. Acad. Sci. U.S.A. 111, 906 (2014).

[20] G. Hodes, Adv. Mater. 19, 639 (2007).

[21] A. Bouhelier, R. Bachelot, G. Lerondel, S. Kostcheev, P. Royer, and G. P. Wiederrecht, Phys. Rev. Lett. 95, 267405 (2005).

[22] R. Lässer, N. V. Smith, and R. L. Benbow, Phys. Rev. B 24, 1895 (1981).

[23] C. Kittel, Introduction to solid state physics, John Wiley \& Sons, 7th edition (1996).

[24] See Supplemental Material at http://link.aps.org/supplemental/... for a detailed explanation of the model of light generation by luminescent intraband relaxation, hot-spot temperature extraction by a direct fit of the black-body spectrum, the variation of the thermal parameter $a$ and effective termparture between hot spots, and the effect of ensemble averaging.

[25] J. T. Hugall, J. J. Baumberg, Nano Lett. 15, 2600 (2015)

[26] G. V. Hartland, Chem. Rev. 111, 3858 (2011). 
[27] N. J. Hogan, A. S. Urban, C. Ayala-Orozco, A. Pimpinelli, P. Nordlander, and N. J. Halas, Nano Lett. 14, 4640 (2014).

[28] A. Manjavacas, J. G. Liu, V. Kulkarni, and P. Nordlander, ACS Nano 8, 7630 (2014). 


\section{Figures and Captions}

\section{Figure 1}
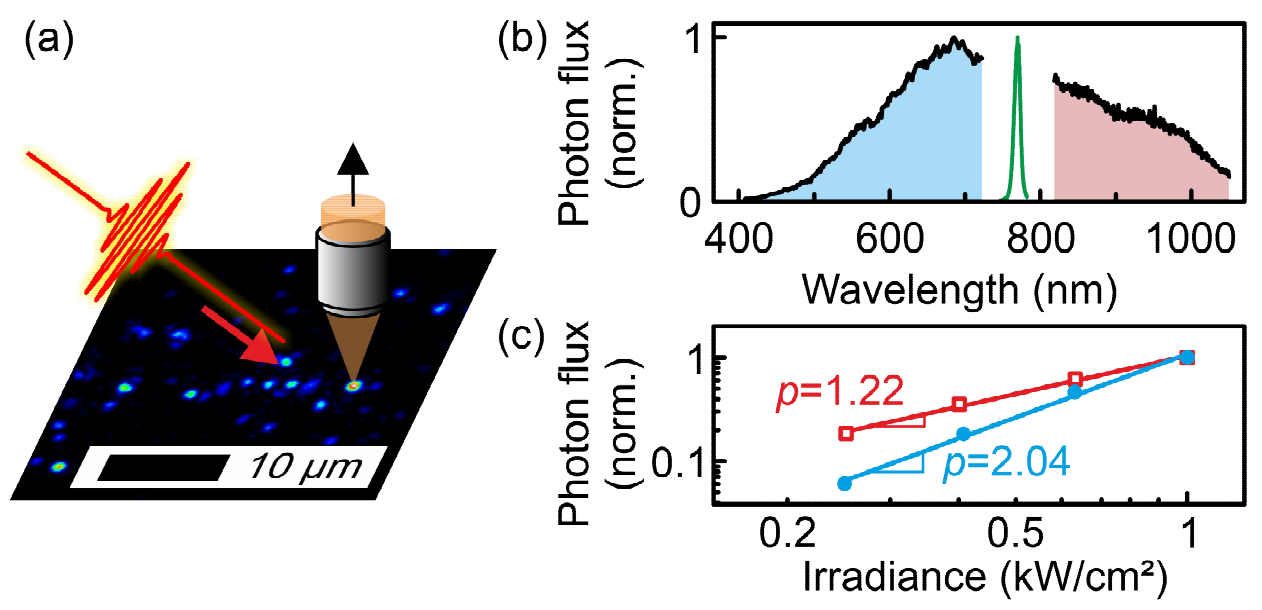

Fig. 1: Localized continuum emission from a single hot spot in a disordered silver nanoparticle film grown by the Tollens reaction, excited by $86 \mathrm{fs}$ laser pulses at $770 \mathrm{~nm}$ wavelength and $80 \mathrm{MHz}$ repetition rate. a) Microscope image of the emission, detected in the $818-1050 \mathrm{~nm}$ spectral range. b) Typical emission spectrum (black) of a single luminescent site for an irradiance of $1 \mathrm{~kW} / \mathrm{cm}^{2}$ with scattered laser radiation (shown in green) blocked by a filter. c) Irradiance dependence of integrated visible (blue) and infrared (red) emission, with power-law exponent $p$ stated. 


\section{Figure 2}

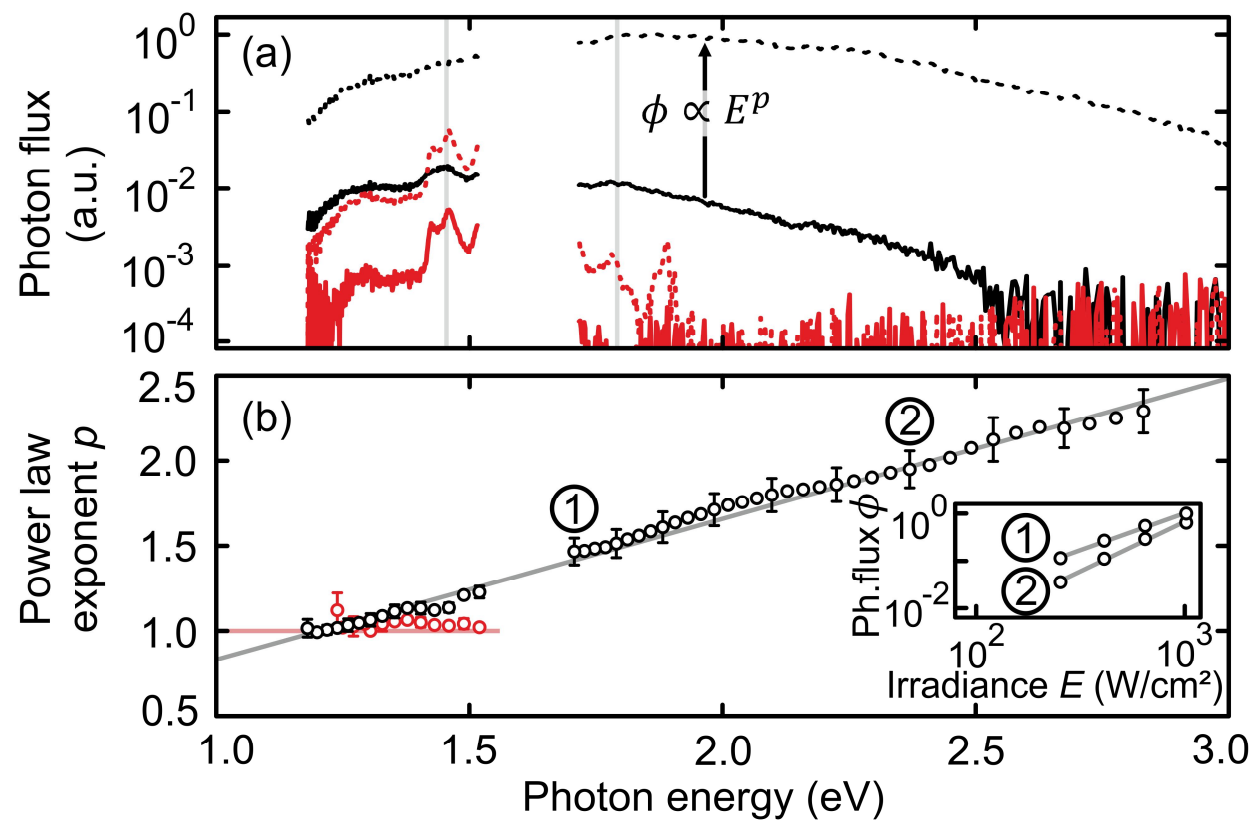

Fig. 2: Spectroscopy of a single luminescent site in a silver nanoparticle film, excited at 770nm $(1.61 \mathrm{eV})$ by either $86 \mathrm{fs}$ laser pulses at $80 \mathrm{MHz}$ repetition rate (black), or continuous-wave illumination (red). (a) Emission spectra at $100 \mathrm{~W} / \mathrm{cm}^{2}$ (solid) and $1 \mathrm{~kW} / \mathrm{cm}^{2}$ (dashed) irradiance, filtered in the range $1.52-1.72 \mathrm{eV}$ to suppress elastically scattered light. Raman bands around $\pm 170 \mathrm{meV}$ relative to the excitation are marked by gray vertical lines. (b) Spectrally resolved power-law exponent for the irradiance dependence of the emitted photon flux, with fit errors indicated for selected data points. The same irradiance range was used for all photon energies. The gray guideline crosses the origin. Two representative fits, marked 1 and 2, are shown in the inset. 


\section{Figure 3}

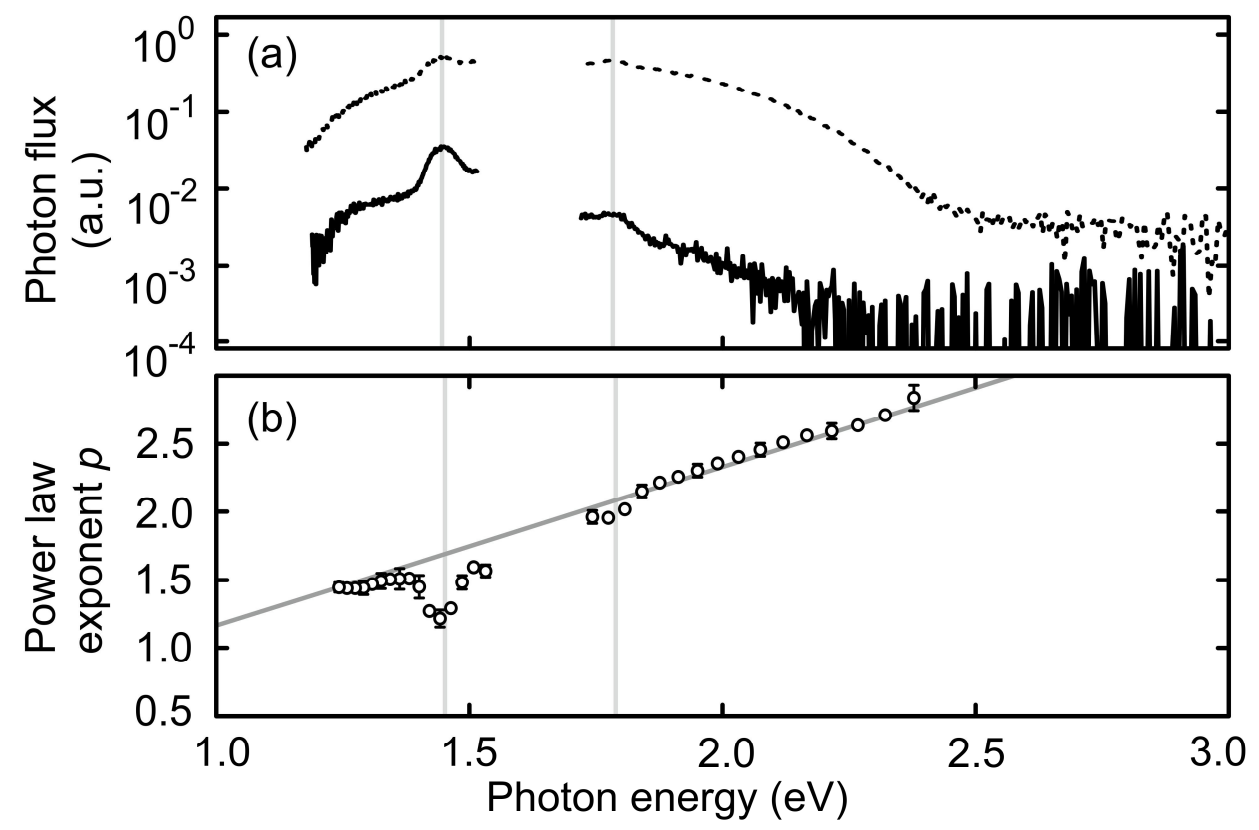

Fig. 3: Non-linearity analysis for a single luminescent hot spot on an evaporated gold film of nominal thickness $\sim 40 \mathrm{~nm}$. (a) Emission spectra at $100 \mathrm{~W} / \mathrm{cm}^{2}$ (solid) and $1 \mathrm{~kW} / \mathrm{cm}^{2}$ (dashed) irradiance with $86 \mathrm{fs}$ pulses at $770 \mathrm{~nm}(1.61 \mathrm{eV})$. Raman bands around $\pm 170 \mathrm{meV}$ relative to the excitation are marked by gray vertical lines. (b) Spectrally resolved power-law exponent. The gray diagonal is a guideline through the origin. 


\section{Figure 4}
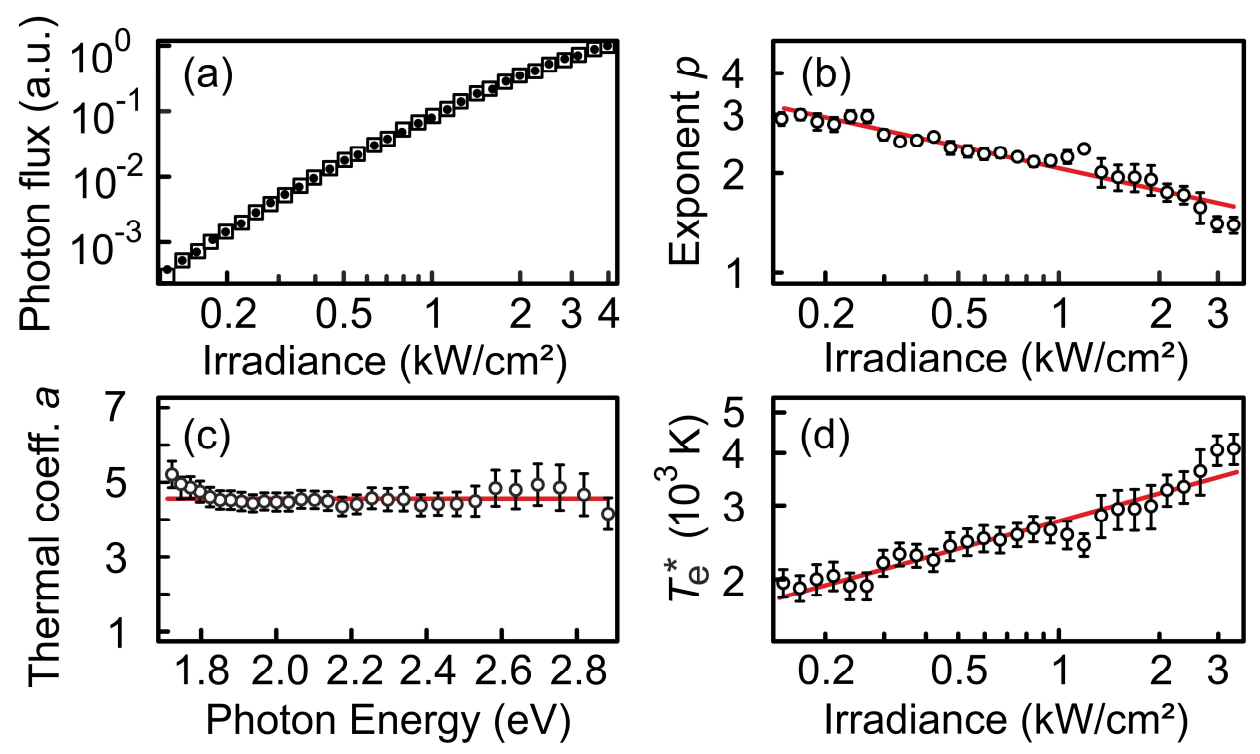

Fig. 4: Extraction of effective temperature $T_{\mathrm{e}}^{*}$ of the electron gas from the irradiance dependence of single hot spot emission in silver nanoparticle films. (a) Logarithmic plot of emission photon flux versus irradiance for an emission window of $2.25 \pm 0.04 \mathrm{eV}$. Circles and squares show two consecutive sweeps in laser power. (b) Logarithmic plot of the irradiance dependence of power-law exponents calculated from the slope of the data in panel (a). A linear fit (red) determines the thermal coefficient $a$. (c) Fit results for coefficient $a$ as a function of the energy of emitted photons. The weighted mean of 4.57 is indicated in red. (d) Electron gas temperature $T_{\mathrm{e}}^{*}$ calculated from the experimental data as a function of irradiance $E^{*}$. The red curve corresponds to $\left(T_{\mathrm{e}}^{*}\right)^{a} \propto E^{*}$ using the average $a=4.57$. 


\section{Word Count Summary}

Text word count

$2630-4+86+113+61+126=3012$

Equations word count

32

Figure word equivalents [(150*height/width $)+20$ words]

Figure $1 \quad$ w. equ. 85

Figure 2 w. equ. 118

$\begin{array}{lll}\text { Figure } 3 & \text { w. equ. } & 118\end{array}$

Figure $4 \quad$ w. equ. $\quad 126$

Tables word equivalent

0

Total

3491 